\title{
Dynamic Current Focusing: A Novel Approach to Loudness Coding in Cochlear Implants
}

\author{
Monique A. M. de Jong, ${ }^{1}$ Jeroen J. Briaire, ${ }^{1}$ and Johan H. M. Frijns ${ }^{1,2}$
}

\begin{abstract}
Objectives: In an attempt to improve spectral resolution and speech intelligibility, several current focusing methods have been proposed to increase spatial selectivity by decreasing intracochlear current spread. For example, tripolar stimulation administers current to a central electrode and uses the two flanking electrodes as the return pathway, creating a narrower intracochlear electrical field and hence increases spectral resolution when compared with monopolar (MP) stimulation. However, more current is required, and in some patients, specifically the ones with high electrode impedances, full loudness growth cannot be supported because of compliance limits. The present study describes and analyses a new loudness encoding approach that uses tripolar stimulation near threshold and gradually broadens the excitation (by decreasing compensation coefficient $\sigma$ ) to increase loudness without the need to increase overall current. It is hypothesized that this dynamic current focusing (DCF) strategy increases spatial selectivity, especially at lower loudness levels, while maintaining maximum selectivity at higher loudness levels, without reaching compliance limits.
\end{abstract}

Design: Eleven adult cochlear implant recipients with postlingual hearing loss, with at least 9 months of experience with their HiRes90K implant, were selected to participate in this study. Baseline performance regarding speech intelligibility in noise (Dutch matrix sentence test), spectral ripple discrimination at 45 and $65 \mathrm{~dB}$, and temporal modulation detection thresholds were assessed using their own clinical program, fitted on a Harmony processor. Subsequently, the DCF strategy was fitted on a research Harmony processor. Threshold levels were determined with $\sigma$ $=0.8$, which means $80 \%$ of current is returned to the flanking electrodes and the remaining $20 \%$ to the extracochlear ground electrode. Instead of increasing overall pulse magnitude, $\sigma$ was decreased to determine most comfortable loudness. After 2 to $3 \mathrm{hr}$ of adaptation to the research strategy, the same psychophysical measures were taken.

Results: At $45 \mathrm{~dB}$, average spectral ripple scores improved significantly from 2.4 ripples per octave with their clinical program to 3.74 ripples per octave with the DCF strategy $(p=0.016)$. Eight out of 11 participants had an improved spectral resolution at $65 \mathrm{~dB}$. Nevertheless, no significant difference between DCF and MP was observed at higher presentation levels. Both speech-in-noise and temporal modulation detection thresholds were equal for MP and DCF strategies. Subjectively, 2 participants preferred the DCF strategy over their own clinical program, 2 preferred their own strategy, while the majority of the participants had no preference. Battery life was decreased and ranged from 1.5 to $4 \mathrm{hr}$.

Conclusions: The DCF strategy gives better spectral resolution, at lower loudness levels, but equal performance on speech tests. These outcomes warrant for a longer adaptation period to study long-term outcomes and evaluate if the outcomes in the ripple tests transfer to the speech scores. Further research, for example, with respect to fitting rules and reduction of power consumption, is necessary to make the DCF strategy suitable for routine clinical application.

Key words: Cochlear implant; Current focusing; Loudness encoding; Spectral resolution; Tripolar stimulation.

(Ear \& Hearing 2018;XX;00-00)

${ }^{1}$ ENT Department, Leiden University Medical Centre, Leiden, The Netherlands; and ${ }^{2}$ Leiden Institute for Brain and Cognition, Leiden University, Leiden, The Netherlands.

\section{INTRODUCTION}

Although average speech understanding has improved in cochlear implant (CI) users in recent decades due to improved CI technology, patients who are implanted with the same device show large variability in speech understanding (Lazard et al. 2012). This is, at least in part, due to differences in the abilities of the CI users to resolve spectral contrast, also termed "spectral resolution." Spectral resolution can be measured with spectral ripple tests, like the recently developed spectral-temporally modulated ripple test (SMRT) (Aronoff \& Landsberger 2013). Several studies revealed that performance on the SMRT is correlated with speech understanding, specifically in difficult listening conditions (Holden et al. 2016; Lawler et al. 2017; Zhou 2017). Moreover, this relation between spectral resolution and speech perception in noise seems to hold across different spectral ripple tests (Henry \& Turner 2003; Litvak et al. 2007b; Won et al. 2007).

Spectral resolution can be limited by poor spatial selectivity, the degree of spread of neural activity across cochlear place (Azadpour \& McKay 2012). Spatial selectivity is influenced by the electrode-neuron interface, that is, by how individual electrode contacts interact with the auditory nerve (Jones et al. 2013). Notably, there is large variability in measures of spatial selectivity, both between and within subjects (Bierer \& Faulkner 2010). Two components of the electrode-neuron interface are thought to underlie these intersubject differences: (1) the electrode-to-neuron distance and (2) spiral ganglion survival (Bierer 2010; Bierer et al. 2015). The distance between electrode contacts and their corresponding neurons is influenced by the electrode design, the surgical placement of the implant (Wardrop et al. 2005a; Wardrop et al. 2005b), the insertion depth (Finley \& Skinner 2008), and bone and tissue growth within the cochlea. Spiral ganglion cell count is determined mainly by the underlying cause and duration of the hearing loss (Nadol et al. 1989).

CIs have multiple electrode contacts along the scala tympani, and each electrode is potentially capable of electrically stimulating a different subpopulation of the surviving auditory neurons in the cochlea. These contacts are usually stimulated in so-called monopolar (MP) mode in which the current is returned to a far-field electrode contact. As a result, the electrical potential field patterns are broad. This causes neighboring electrodes to activate overlapping populations of neurons, especially if the electrode-to-neuron distance is substantial, because this decreases spatial selectivity and reduces the number of spectral channels that can be distinguished (Bierer 2010). Poor spiral ganglion survival can be addressed in part by increasing the current amplitude, although this, in turn, increases the current spread and can therefore exacerbate the issue described earlier.

In an attempt to improve spatial selectivity, and therefore spectral resolution and speech intelligibility, several current 
focusing methods have been proposed that increase spatial selectivity by reducing intracochlear current spread. Computer modeling data (Kalkman et al. 2015), as well as animal (Kral et al. 1998) and human (Bierer 2007; Berenstein et al. 2008; Bierer 2010; Landsberger et al. 2012; Zhu et al. 2012; Smith et al. 2013; Padilla \& Landsberger 2016) data, show that current focusing creates a narrower intracochlear electrical field and hence increases spectral resolution compared with MP stimulation. For example, tripolar (TP) stimulation administers current to a central electrode and uses two flanking electrodes as the return pathways. This current focusing strategy improves speech understanding, with some researchers suggesting that especially poor performers would benefit from TP stimulation (Srinivasan et al. 2012; Bierer \& Litvak 2016). However, this gain comes at the expense of an increased amount of current required to achieve a given loudness. In some patients, specifically in patients with high electrode impedances, only part of the dynamic range can be covered within the compliance limit of the implant (Kral et al. 1998; Mens \& Berenstein 2005; Bierer 2007). Moreover, because the stimulus level partly determines the current spread, the benefit in the spectral domain may be compromised at higher loudness levels (Khalili Moghaddam et al. 2014).

The problem of limited loudness growth was addressed by the introduction of a partial TP (pTP) strategy in which only a fraction $\sigma$ (called the compensation coefficient) of the current is returned to the flanking electrodes (Mens \& Berenstein 2005; Berenstein et al. 2008). In fact, this strategy uses a superposition of the MP and TP stimulation strategies. Relative to the TP strategy, the pTP strategy results in greater loudness at the expense of less selective stimulation. Despite the reduced level of current focusing, pTP stimulation improves spectral ripple discrimination (Berenstein et al. 2008; Smith et al. 2013), while speech perception showed to be improved in some (Srinivasan et al. 2013; Wu \& Luo 2013), but not in all studies (Berenstein et al. 2008; Bierer \& Litvak 2016). Nogueira et al. (2017) developed a stimulation mode called the dynamically compensated virtual channel in which four adjacent electrodes are stimulated simultaneously to decrease power consumption. Although this quadrupolar strategy saves power, it also generates broader electrical fields, specifically at higher loudness levels. To compensate for this, current focusing is applied by sending current of opposite polarity to the two outer electrode contacts. Loudness balancing experiments with different degrees of current focusing revealed that higher degrees of current focusing result in significantly higher current levels that are required to maintain equal loudness.

The present study describes and analyses a new approach to loudness encoding that is called "dynamic current focusing" (DCF). Previous research showed that loudness is increased at fixed current levels by lowering the degree of current focusing (Vellinga et al. 2017a). The DCF uses pTP stimulation near the threshold, and it gradually broadens the excitation by decreasing the compensation coefficient $\sigma$ to increase loudness without the need to increase the overall current (see Fig. 1 for a schematic overview). In practice, this means that more current is consumed at low than at high loudness levels, because the current levels for the flanking electrodes are lowered with increasing loudness, while the level on the center contact remains the same. It is hypothesized that the DCF strategy increases spatial selectivity, especially at lower loudness levels, while maintaining the most
Dynamic Current Focusing Monopolar stimulation

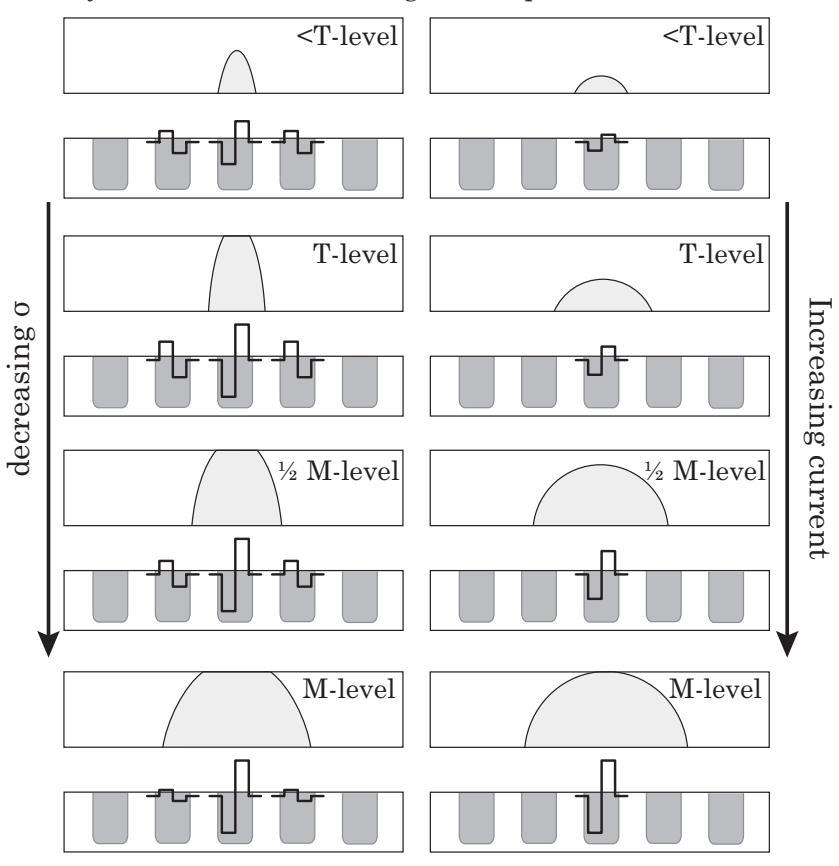

Fig. 1. The concept of loudness coding in the DCF strategy and in MP stimulation mode. The upper bar for each loudness step shows the auditory nerve with the excitation shown pattern in gray. The lower bars show the implanted electrode array with the electrode contacts in gray. In the DCF strategy, the amplitudes of the main and neighboring electrode contacts are increased equally up to the T-level. To increase the loudness from the T-level, $\sigma$ is decreased as a function of the stimulus level, resulting in a broader excitation pattern and, accordingly, in a higher loudness level. In MP mode, the amplitude of the main electrode contact is increased as a function of the stimulus level, resulting in broad current spreads at all loudness levels. DCF indicates dynamic current focusing; M-level, most comfortable level; MP, monopolar; T-level, threshold level.

optimal selectivity possible at higher loudness levels and staying within device compliance limits. This optimal spatial selectivity across the dynamic range would then lead to an improved spectral resolution, primarily at lower loudness levels. Here, we evaluated the novel DCF loudness coding strategy in 11 subjects in an acute setting in which all tasks were performed with both their conventional clinical strategy and the DCF strategy on a single day. Spectral resolution was assessed with the SMRT, which measures the spectral ripple density threshold. In addition, temporal modulation detection thresholds (MDTs), speech intelligibility in noise, and loudness growth functions were assessed.

The study was approved by the Medical Ethical Committee of the Leiden University Medical Center (ref. P02.106.AA).

\section{MATERIALS AND METHODS}

\section{Listeners}

Eleven adults with postlingual hearing loss who were unilaterally implanted with an Advanced Bionics (HiRes90K, Sylmar, CA) implant for at least 9 months were selected to participate in this study. None of the subjects had functional hearing in the contralateral ear. Because the DCF strategy uses multipolar stimulation, only CI users with all 16 electrode contacts working were included in this study. The study included 4 women and 7 men 48 to 79 years of age. Of these, 1 subject used HiResolution (HiRes, Sylmar, CA) (Firszt 2003) in the 
clinical setting, 4 subjects used HiRes Fidelity 120 (HiResF120, Sylmar, CA) (Büchner et al. 2012), and 6 subjects used HiRes Optima, Sylmar, CA (Advanced Bionics, Sylmar, CA 2012). Table 1 shows the patients' clinical characteristics.

After the assessment of baseline performance with their clinical strategy fitted on a dedicated Harmony sound processor in the laboratory, the DCF strategy was fitted on this research processor. The subjects had 2 to $3 \mathrm{hr}$ to adjust to the experimental strategy by taking a break in a busy restaurant, during which they were actively communicating with the researcher. After this, their performance with DCF was evaluated on the same day. The only exception was S01, who was unable to perform all psychophysical tasks in a single day due to fatigue. For this subject, the DCF strategy was evaluated after 10 extra minutes of adaptation on a separate test day. The subjects were not blinded to the tested speech coding strategy as the subjects could easily detect their normal strategy, and the order of the tested strategy was not randomized.

\section{Fitting Procedures}

Clinical Strategy - The data from each subject's last clinical visit were copied from the SoundWave program (Advanced Bionics, Valencia, CA) and fitted on a Harmony research processor. If the noise cancelation features were active in their every day program, they were turned off for the testing.

Research Strategy - The concept of the DCF strategy is schematically displayed and compared with MP stimulation in Figure 1. The DCF program was created for each subject using BEPS+ software (Advanced Bionics, Valencia, CA). Threshold levels (T-levels) were determined for each electrode contact by gently increasing the total amount of current at $\sigma=0.8$, meaning that $80 \%$ of the current is returned equally to the two flanking electrodes and $20 \%$ is returned to the extracochlear electrode. To ensure that sufficient loudness is achieved if $\sigma$ is reduced to zero (i.e., MP stimulation on the central electrode contact), current on the central electrode contact had to be at least 300 clinical units (CUs). CUs represent constant charge, which means that automatic adjustments in pulse width also result in automatic adjustments in pulse amplitude to maintain constant charge: [ amplitude (in A) p pulsewidth (in sec) $k$ (scaling constant) ]. A level of 300 CUs was chosen because in the clinical fittings (in MP stimulation mode), all subjects' most comfortable level (M-level) values were below $300 \mathrm{CUs}$. It was therefore expected that the full dynamic range could be covered by varying the compensation coefficient $\sigma$, and not increasing the current level on the center electrode contact above 300 CUs. If the total current necessary for the T-level was below 300 CUs at $\sigma=0.8$, the T-level was determined again, using a compensation coefficient $\sigma=0.9$. If total current was still below $300 \mathrm{CUs}$, T-levels were determined using $\sigma=1.0$. The used $\sigma$ values at T-level are depicted in Table 1. Next, the M-levels for the DCF strategy were determined by gradually decreasing $\sigma$ in steps of 0.01 , while the current levels on the central electrode contacts were kept constant, thereby broadening the excitation pattern. As a result, the dynamic range is defined by variations in $\sigma$ and the subjective loudness at T- and M-levels is perceived similar to that with their clinical strategy. To verify the latter, subjects were asked if the loudness level with the speech program turned on was similar to that with their regular strategy. If this was not the case, the loudness level was adjusted accordingly. No loudness

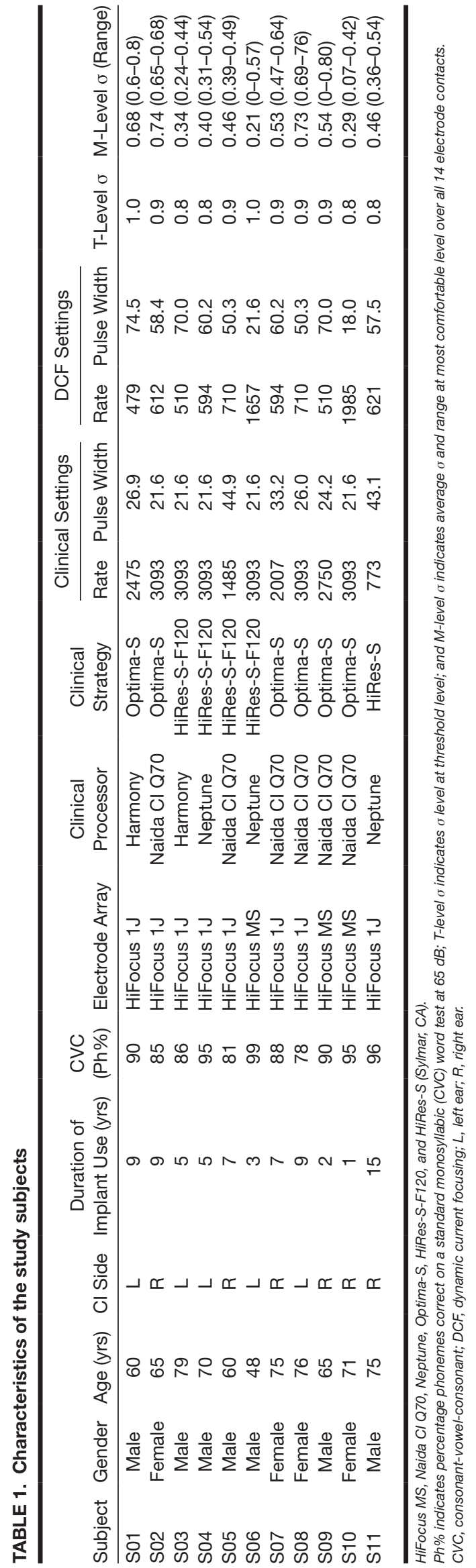


balancing per electrode contact was applied. Low power modes (e.g., automated power management and reduced maximum power mode) were turned off to avoid potential difficulties with power management, as well as noise reduction algorithms. Because three physical electrode contacts are required to create one current focusing channel, the two outer electrodes could not be used. Therefore, the DCF strategy had only 14 effective channels while the clinical strategy had 16 (HiRes) or 120 theoretical (current steering) spectral channels.

\section{PSYCHOPHYSICAL TASKS}

\section{Loudness Growth Functions}

For both stimulation strategies, a loudness scaling experiment was performed at three different locations along the electrode array (electrodes 3, 9, and 14). Because the two stimulation strategies use different mechanisms to achieve M-level, it is impossible to compare them in the same quantity when the electrode contacts are directly stimulated. In MP stimulation, the driving factor to achieve M-level is current, while there is not one individual current or electrode contact to which you can link loudness growth in DCF stimulation. Therefore, the "acoustic" stimuli, that were generated using a custom MATLAB program (MathWorks Inc., Natick, MA), were presented via a direct input system into the Harmony speech processor. In this way, the same stimuli (sine waves with frequencies corresponding to the center frequencies of electrode 3, 9, or 14, and with amplitude V) could be generated for the two stimulation strategies, after which they were processed with the use of either the MP or DCF speech coding strategy. The stimulus levels were calculated as follows:

$$
\text { Loudness Level }(\text { in } \mathrm{dB})=20 \cdot \log _{10}\left(\frac{V}{V_{\text {ref }}}\right)
$$

with $V_{r e f}=10 \mu \mathrm{V}$. The level was slowly increased in step sizes of 5 $\mathrm{dB}$, starting from $0 \mathrm{~dB}$ and never exceeding $100 \mathrm{~dB}$ to avoid overstimulation. Loudness was subjectively rated on an eight-point loudness scale as used in our previous current focusing experiments (Vellinga et al. 2017a). This loudness scale ranged from the T-level (1), to the most comfortable loudness (5) to the upper limit of comfortable loudness (8); after this, the experiment was terminated (Potts et al. 2007). The experiment was repeated three times. To quantify the slope of the loudness scaling curves, the areas under the curves (AUCs) were calculated as follows:

$$
\mathrm{AUC}=\sum_{i=2}^{4} \Delta \mathrm{LL}_{i} \frac{\mathrm{SL}_{i}+\mathrm{SL}_{i+1}-4}{2}
$$

with $i=$ subjective loudness level, which was ranging from 2 to 5 (from "very soft sound" to "most comfortable loudness") as these levels were considered to be the most important for understanding speech. $\mathrm{SL}=$ subjective loudness, $\Delta \mathrm{LL}=$ difference in loudness level (in $\mathrm{dB}$ ) between $\mathrm{SL}_{i}$ and $\mathrm{SL}_{i+1}$. Differences in AUCs ( $\triangle \mathrm{AUC}$ ) between the two strategies were expressed as a percentage relative to the AUC for the clinical strategy $\left(\mathrm{AUC}_{\text {clinical }}\right)$ :

$$
\Delta \mathrm{AUC}[\%]=\frac{\mathrm{AUC}_{\mathrm{DCF}}-\mathrm{AUC}_{\text {clinical }}}{\mathrm{AUC}_{\text {clinical }}} \cdot 100 \%
$$

with $\mathrm{AUC}_{\mathrm{DCF}}=$ the AUC for the DCF strategy. The offset of the loudness growth functions was measured at the level (in $\mathrm{dB}$ ) at subjective loudness level 2 for each electrode contact and stimulation strategy.

$$
\Delta \text { offset }[\mathrm{dB}]=\sum_{e \in S}\left(\text { offset }_{\mathrm{DCF} e}-\text { offset }_{\text {clinical } e}\right) / 3
$$

with $S=3,9$, or 14 , referring to electrode contacts $(e)$, and where $\Delta$ offset was calculated per stimulation strategy as the average difference in offset of the three electrode contacts.

Because of the pilot-like nature of this study, the research protocol was fine tuned during the trial. Accordingly, the first 3 subjects ( $\mathrm{S} 01, \mathrm{~S} 02$, and S03) had a slightly different research set up than subsequent subjects. For these subjects, loudness curves were obtained as a function of the current in microampere for the clinical strategy and as a function of $\sigma$ in the DCF strategy, using a direct connection to the implant. Because this made it impossible to compare the loudness growth functions between the strategies, we switched to the direct connection to the speech processer described earlier, and discarded the loudness scaling data for the first 3 subjects.

\section{Psychophysical Tasks-Sound Booth Testing}

The tasks described later were performed in the free field, with subjects seated $1 \mathrm{~m}$ away from the front of a single loudspeaker in a double-walled sound-attenuating booth. To minimize the impact of learning effects on the results of the psychophysical tasks, the subjects went through a dry run before each actual test run.

\section{Spectral Ripple Test}

The SMRT (Aronoff \& Landsberger 2013) was used to determine spectral ripple thresholds at 45 and $65 \mathrm{~dB}$. It is a three-alternative forced choice task that determines the maximum ripples per octave (RPO) that a listener can differentiate from two reference stimuli that have ripple densities of 20 RPO. Study subjects were asked to indicate the deviant stimulus from the three sounds without receiving feedback about the correct answer. This spectral ripple density test was chosen because, as opposite to the previously existing spectral ripple test (e.g., Henry \& Turner 2003; Won et al. 2007), the SMRT was designed to avoid a number of potential confounders, like cues that are related to local loudness or the spectral center of gravity (Aronoff \& Landsberger 2013). Moreover, the SMRT has been shown to correlate with speech recognition in noise in a variety of CI users (Lawler et al. 2017). The procedure was repeated six times per condition, and the average thresholds were calculated.

\section{Temporal Modulation Detection Test}

Temporal resolution was assessed with the temporal modulation detection test, adapted from Won et al. (2011). The two alternative adaptive measures have two wideband noise stimuli: one without amplitude modulation and one with a modulation frequency of $100 \mathrm{~Hz}$ that was adaptive in modulation depth. A modulation frequency of $100 \mathrm{~Hz}$ was chosen because this task, along with spectral ripple thresholds, accounts for the highest amount of variance in consonant-vowel-consonant word scores (Won et al. 2011). The subjects were asked to identify the interval that contained the modulated noise and were then given feedback about whether this was the correct answer. The task was performed at $65 \mathrm{~dB}$ and repeated six times per condition, 
then the average MDTs were calculated in $\mathrm{dB}$ relative to $100 \%$ modulation $\left(20 \cdot \log _{10}\right.$ modulation depth $)$.

\section{Speech-in-Noise Test}

The Dutch matrix sentence test is an adaptive speech-innoise test that uses 50 unique words that are combined into 200 grammatically equivalent sentences, which are grouped into 10 balanced lists (Houben et al. 2014). At each test round, the sentences are randomly selected from the subset. The task was carried out using the APEX 3 program (Leuven, Belgium) (Francart et al. 2008), installed on a personal computer. After the presentation of a sentence, the subjects are asked to repeat the five words and to guess if they are not sure. Testing was done at a fixed speech level of $65 \mathrm{~dB}(\mathrm{~A})$ and with the adaptive speech-shaped noise starting from $-4 \mathrm{~dB}$ signal to noise ratio (SNR). The outcome measure of the matrix test is the speech reception threshold (SRT), which is defined as the SNR at which $50 \%$ of the words are repeated correctly. An average SNR was calculated over three repetitions to determine the final SRT score. During the matrix task practice session, subjects were exposed to all possible words.

\section{Subjective Rating}

The subjective quality of the incoming sound in terms of overall loudness, loudness growth, sound clarity, speech understanding, etc. was discussed with the subjects. In addition, all subjects were asked whether they would be able to function normally with this new program in their home situation and if their overall rating of the DCF strategy was better, equal to, or worse than their clinical program.

\section{Statistical Analysis}

Repeated measurements were obtained in all experiments; therefore, two-way repeated-measures analysis of variance (ANOVA) was used for the statistical analysis. The factors "speech coding strategy" (clinical and DCF) and "repetition number" ( 1 to 3 for the speech-in-noise test and 1 to 6 for the spectral ripple and the temporal modulation detection test) were used to determine if there was a main effect of the speech coding strategy, repetition number, and interaction between the two factors. IBM SPSS Statistics for Windows (Armonk, NY), Version 23.0, was used for calculations.

\section{RESULTS}

\section{Loudness Growth Functions}

The individual loudness growth functions of S04 to S11 were obtained for the clinical and the DCF strategies. The loudness scores are plotted as a function of the presented stimulus level in $\mathrm{dB}$ (Fig. 2). The AUCs are depicted by the filled AUCs. Both the slope (expressed in AUC) and the offset of the

Electrode 3 Electrode $9 \quad$ Electrode 14
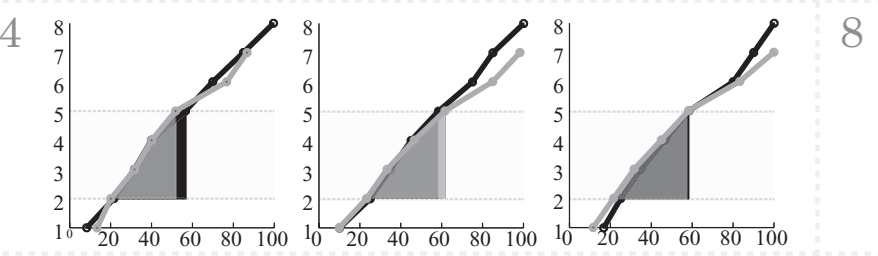

Electrode 3

Electrode 9

Electrode 14
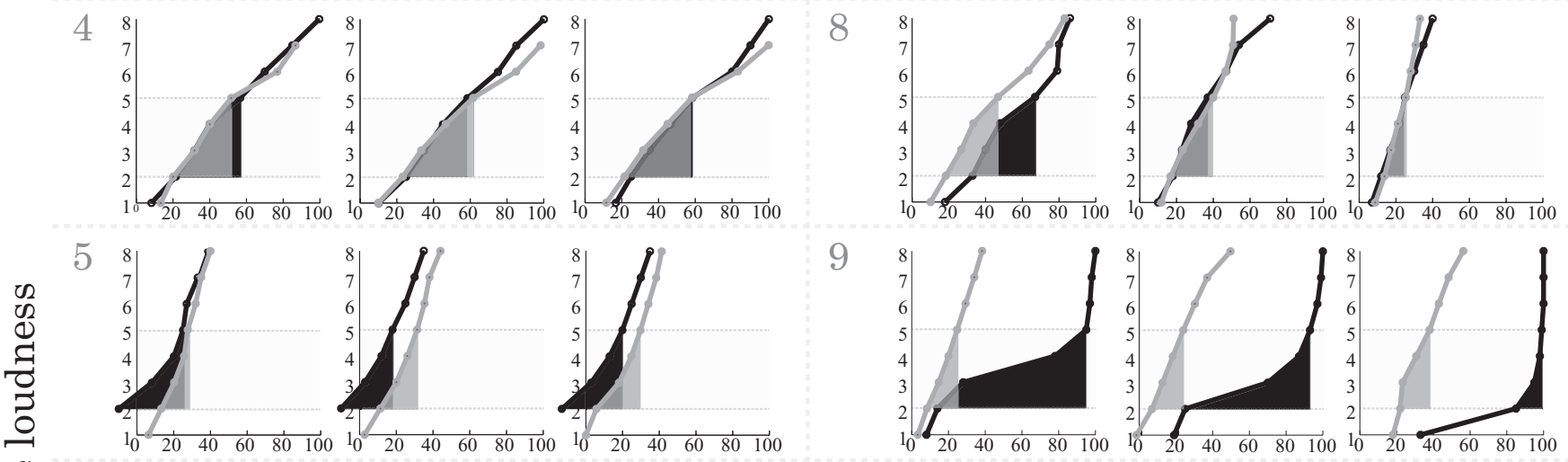

9
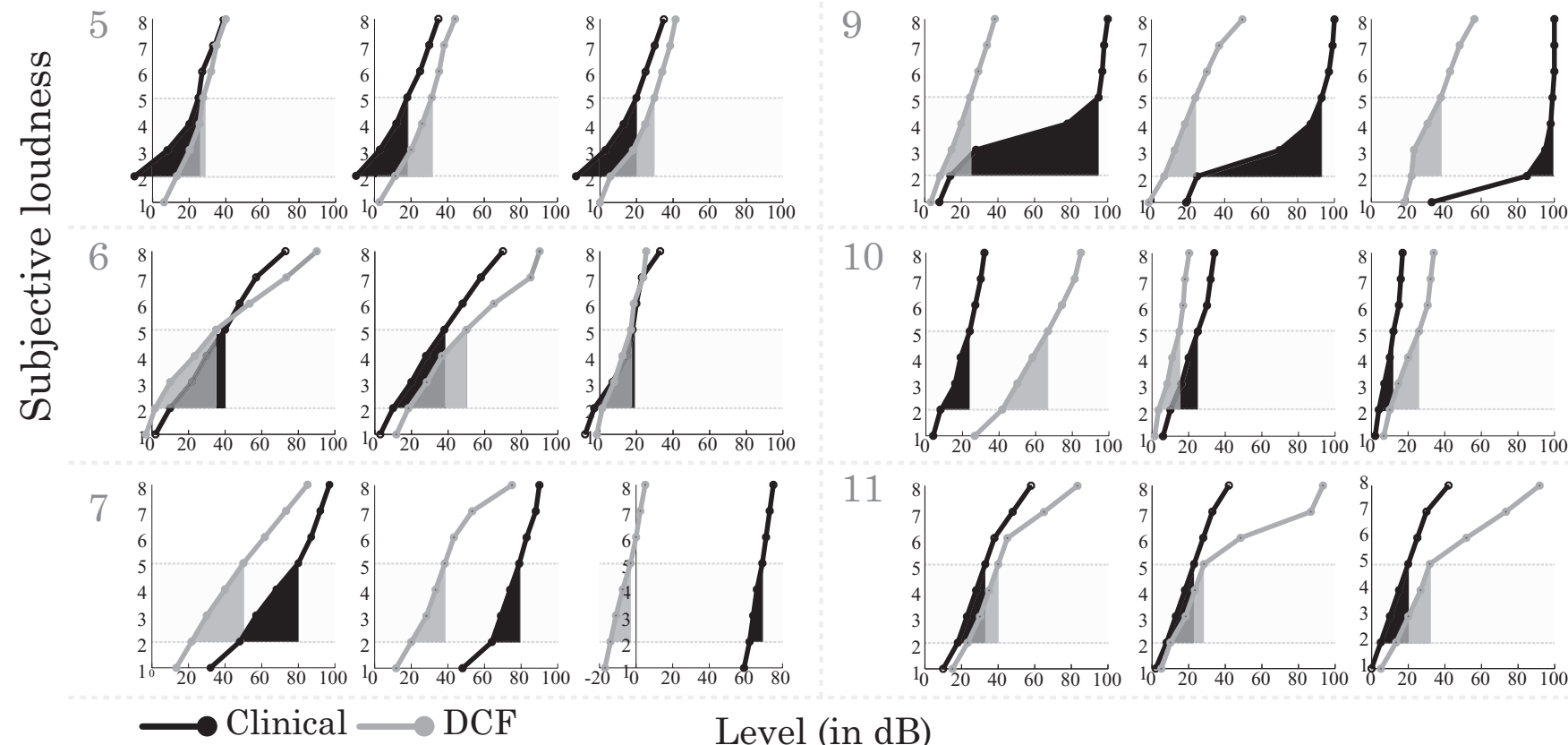

11
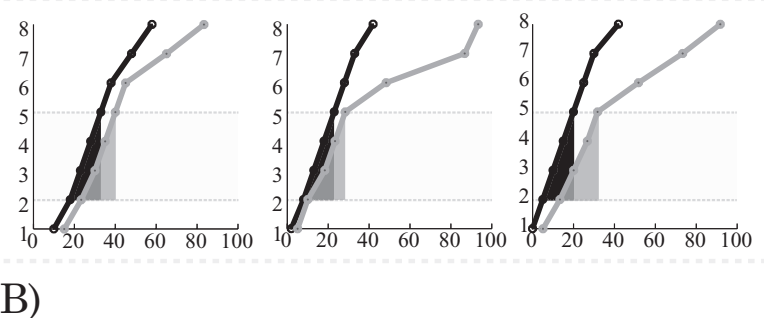

Fig. 2. The individual loudness growth functions of S04 to S11 on an eight-point loudness scale (y axes). The stimulus levels were calculated as follows: Loudness Level $($ in $\mathrm{dB})=20 \cdot \log _{10}\left(V / V_{\text {ref }}\right)$, with $V_{\text {ref }}=10 \mu \mathrm{V}$. For understanding speech, the most important loudness levels are considered to range from "2" (very soft sound) to " 5 " (the most comfortable loudness). This range is shaded gray. The AUCs are patterned for the two strategies. AUC indicates areas under the curve; DCF, dynamic current focusing. 
TABLE 2. Differences in the area under the loudness curves ( $\triangle A U C)$ between the strategies for each subject at the indicated electrodes

\begin{tabular}{lcccc}
\hline Subject & Electrode 3 (\%) & Electrode 9 (\%) & Electrode 14 (\%) & Absolute Average (\%) \\
\hline S04 & -20 & +17 & +13 & 17 \\
S05 & -58 & -26 & -28 & 37 \\
S06 & +23 & +20 & -8 & 17 \\
S07 & -14 & -10 & +45 & 23 \\
S08 & -23 & -19 & -1 & 14 \\
S09 & -82 & -60 & +161 & 71 \\
S10 & +72 & -26 & +117 & 72 \\
S11 & +4 & +7 & +15 & 9 \\
\hline
\end{tabular}

The (absolute) average $A U C$ of the three electrode contacts is also shown and is expressed as the percentages of the $A U C$ for the $M P$ strategy $\left(\triangle A U C[\%]=A U C_{D C F}-A U C_{c l i n i c a l} / A U C_{\text {clinical }} \cdot 100 \%\right)$. AUC, area under the curve; MP, monopolar.

loudness growth curves with the DCF strategy showed considerable deviations from the corresponding values with the clinical strategy in some subjects. The individual differences in AUCs ( $\triangle$ AUC) per electrode as calculated from the data are presented in Table 2. S05, S09, and S10 showed the largest $\Delta$ AUCs, while the loudness growth functions of S04, S06, S07, S08, and S11 were quite similar in terms of the AUCs. Table 3 shows the differences in offsets ( $\Delta$ offset) of the loudness growth curves per electrode contact, where the offset at loudness level 2 with the clinical program was subtracted from the one with DCF. It turned out that S05, S07, S09, and S10 showed large discrepancies between the offsets with the two strategies, when compared with the other subjects. For S05 and S10, $\Delta$ offset was negative, meaning that for DCF, a higher input was required to reach the offset.

\section{Spectral Ripple Test}

The individual and mean spectral ripple discrimination thresholds are shown in Figure 3. At $45 \mathrm{~dB}$, the mean thresholds for the clinical and the DCF strategies were 2.40 RPO and 3.74 RPO, respectively; at $65 \mathrm{~dB}$, the values were $3.27 \mathrm{RPO}$ and 4.07 RPO, respectively. As shown in Figure 3A, 8 of 11 subjects showed an improved spectral resolution with the DCF strategy relative to their clinical strategy at $45 \mathrm{~dB}$. A two-way repeated-measures ANOVA showed that the average difference was statistically significant at $45 \mathrm{~dB}$ for $1.34 \mathrm{RPO}[F(1,10)=8.369 ; p=0.016]$. No effect was observed for the repetition number $[F(5,50)=0.080 ; p=0.995]$ or for the interaction between strategy and repetition number $[F(5,50)=1.069 ; p=0.389]$. At $65 \mathrm{~dB}$, also 8 of 11 subjects showed improved spectral ripple thresholds (Fig. 3B). No significant difference was observed between DCF and MP at $65 \mathrm{~dB}[F(1,10)=$ $2.186 ; p=0.170]$. This result may be explained by the less focused stimulation provided by DCF at these presentation levels. Further, there was no effect for the repetition number $[F(5,50)=0.227 ; p=$ 0.949 ] or for the interaction between strategy and repetition number [Greenhouse-Geisser corrected $F(2.8,27.7)=2.581 ; p=0.078$ ]. All subjects improved on spectral ripple discrimination at either $45 \mathrm{~dB}$ or at $65 \mathrm{~dB}$ or both. In a post hoc analysis, where the subjects with the largest $\triangle \mathrm{AUC}(\mathrm{S} 05, \mathrm{~S} 09$, and S10) were excluded, there was a highly significant improvement from $2.8 \mathrm{RPO}$ to $4.4 \mathrm{RPO}$ at $65 \mathrm{~dB}$ $[F(1,7)=14.862 ; p=0.006]$. Although there was no significant correlation between the $\triangle \mathrm{AUC}$ and spectral ripple performance at 45 $\mathrm{dB}$ (Fig. 4A), exclusion of the same three AUC outliers resulted in a highly significant improvement from $2.4 \mathrm{RPO}$ to $4.2 \mathrm{RPO}[F(1,7)=$ $11.264 ; p=0.012]$. Linear regression revealed that (when including S05, S09, and S10 in the analysis) the $\triangle \mathrm{AUC}$ could significantly predict spectral ripple performance with the DCF strategy at 65 $\mathrm{dB}[F(1,6)=7.079 ; p=0.037]$. The average $\Delta$ AUC accounted for $54.1 \%$ of the variation in spectral ripple scores with an adjusted $R^{2}$ $=46.5 \%$. The regression equation was as follows:

$$
P_{\mathrm{RPO} \_65 \mathrm{~dB}}=2.25-0.046 \cdot \Delta \mathrm{AUC}
$$

where $P_{\mathrm{RPO} \_65 \mathrm{~dB}}$ is the predicted improvement in RPO with the DCF strategy when compared with the clinical strategy, at 65 dB (Fig. 4B).

When the two outliers that drove the direction of the correlation (S09 and S10) were excluded from the linear regression, however, the correlation completely disappeared $[F(1,4)=0.001 ; p=0.979]$. These subjects drive the correlation because they were poor performers with the DCF strategy and had large differences in their $\triangle \mathrm{AUCs}$. By excluding these subjects from this analysis, only the better performing subjects were included. The differences in the offset of the loudness curves were also analyzed, but the correlation between $\Delta$ offset

TABLE 3. Differences in the offsets ( $\Delta$ offsets) of the loudness curves (loudness level 2 of the loudness scaling procedure) for each subject at the indicated electrodes (clinical - DCF)

\begin{tabular}{lccrr}
\hline Subject & Electrode 3 $(\mathrm{dB})$ & Electrode 9 $(\mathrm{dB})$ & Electrode 14 (dB) & Average $(\mathrm{dB})$ \\
\hline S04 & +1.7 & +1.7 & +3.3 & -19.0 \\
S05 & -23.3 & -21.3 & -4.7 & -2.2 \\
S06 & +8.3 & -8.3 & +75.7 & -1.6 \\
S07 & +25.8 & +44.0 & -2.0 & +48.5 \\
S08 & +15.0 & +1.7 & +63.3 & +5.7 \\
S09 & +5.7 & +17.7 & -5.7 & +28.9 \\
S10 & -33.7 & +6.5 & -8.0 & -11.0 \\
S11 & -5.0 & -1.7 & & -4.9 \\
\hline
\end{tabular}



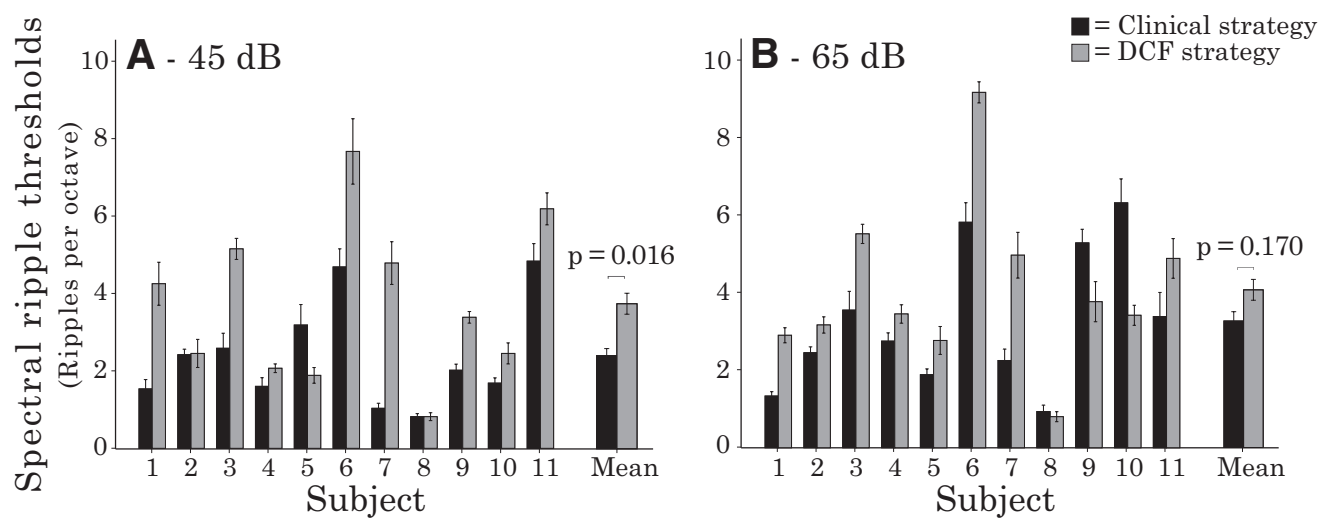

Fig. 3. Individual and mean spectral ripple thresholds at $45 \mathrm{~dB}$ SPL (A) and at $65 \mathrm{~dB}$ SPL (B) for the 11 study subjects using their clinical strategy and the DCF strategy. The error bars represent \pm 1 standard error of the mean. DCF indicates dynamic current focusing.

and spectral ripple performance at $45 \mathrm{~dB}$ was not statistically significant $[F(1,6)=5.6 ; p=0.056)$ (Fig. $4 \mathrm{C}$ ) and also no correlation at $65 \mathrm{~dB}(p=0.65)$ (Fig. 4D) was found.

\section{Temporal Modulation Detection Test}

The individual and mean MDTs in $\mathrm{dB}$ relative to $100 \%$ amplitude modulation, that were measured at comfortable level, are shown in Figure 5. The mean MDTs were $-9.35 \mathrm{~dB}$ for the clinical speech coding strategies and $-8.73 \mathrm{~dB}$ for the DCF strategy. A two-way repeated-measures ANOVA revealed no significant differences in performance between the two speech coding strategies $[F(1,10)=12.611 ; p=0.497]$ in terms of amplitude modulation detection. There were no correlations between the $\triangle \mathrm{AUC}$ or $\Delta$ offset and MDT scores, and the exclusion of the AUC or $\Delta$ offset outliers did not change the MDT results.

\section{Speech-in-Noise Test}

The individual and mean SRTs, as measured at $65 \mathrm{~dB}$, are shown in Figure 6. The mean SRTs were 4.57 and 4.72 dB SNR for the clinical and DCF strategies, respectively. The standard
A

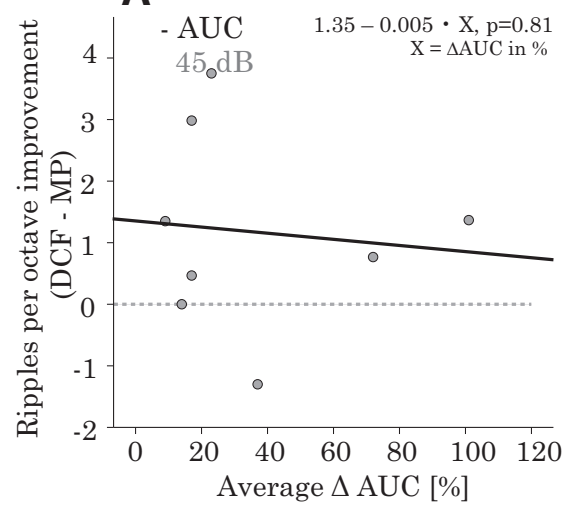

C

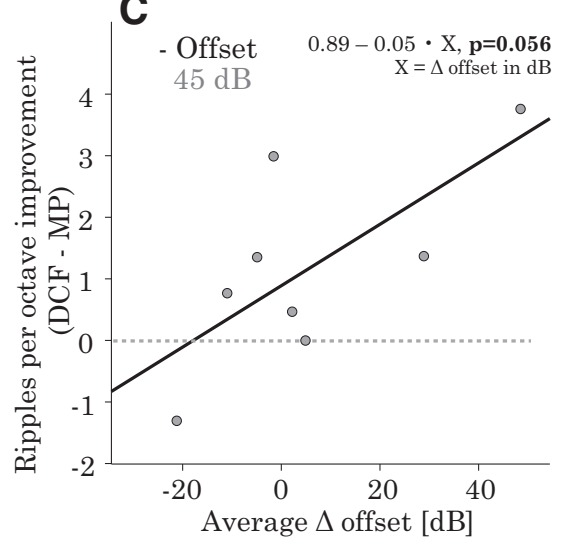

B
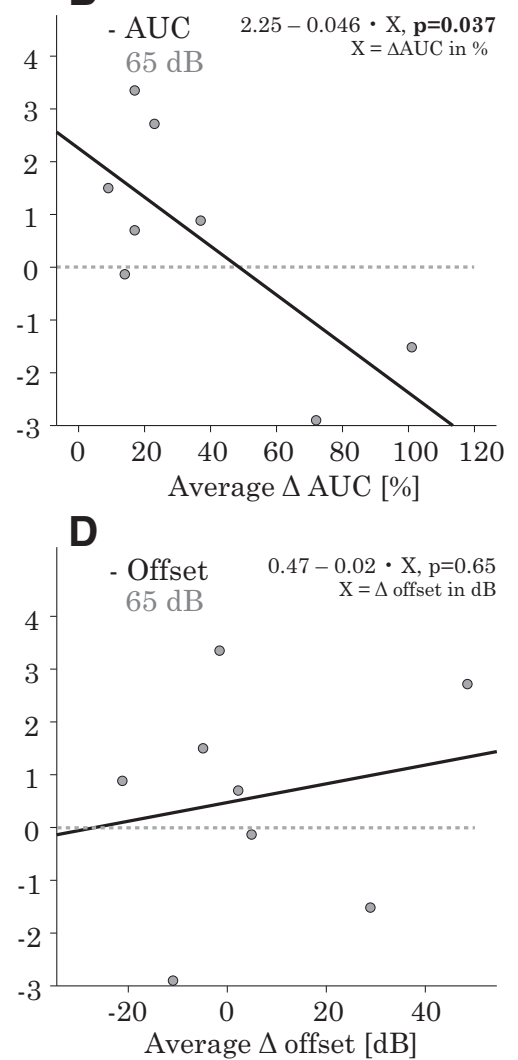

Fig. 4. Correlation between deviating loudness growth and improvement on the spectral ripple task at 45 and $65 \mathrm{~dB}$ SPL. Deviating loudness growth was expressed as the absolute average delta $\mathrm{AUC}$ of the three electrode contacts $\left[\triangle \mathrm{AUC}[\%]=\left(\mathrm{AUC} \mathrm{C}_{\mathrm{DCF}}-\mathrm{A} \cup \mathrm{C}_{\mathrm{clinical}}\right) / \mathrm{AUC} \mathrm{C}_{\mathrm{clinical}} \cdot 100 \%\right](\mathrm{A}$ and $\mathrm{B})$ and the average difference in offset of the loudness growth curves $\left[\Delta\right.$ offset $[\mathrm{dB}]=\sum_{\mathrm{e} \in S}\left(\right.$ offset $_{\mathrm{DCF}}$ - offset $\left.\left._{\text {clinicale }}\right) / 3\right]$ with DCF and the clinical program $(C$ and D). AUC indicates areas under the curve; DCF, dynamic current focusing; MP, monopolar. 


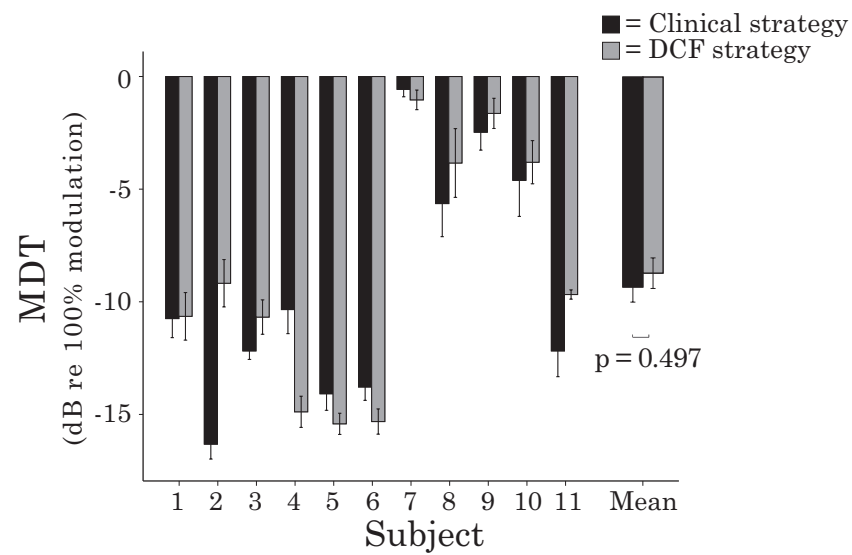

Fig. 5. Individual and mean MDTs for the 11 study subjects at $65 \mathrm{~dB}$ SPL. The error bars represent \pm 1 standard error of the mean. DCF indicates dynamic current focusing; MDT, modulation detection threshold.

errors were quite large for some subjects, and a two-way repeated-measures ANOVA revealed that the difference in SRTs was not statistically significant $[F(1,10)=0.019 ; p=0.893]$. No correlations were found between the $\triangle \mathrm{AUC}$ or $\Delta$ offset and SRT values, and exclusion of the AUC or $\Delta$ offset outliers did not change the speech-in-noise results.

\section{Subjective Rating}

Subjectively, 2 subjects preferred the DCF strategy over their own clinical strategy and 7 had no preference. The remaining 2 subjects preferred their own strategy over the acutely tested DCF research strategy. Some subjects noted that the DCF strategy resulted in a "richer" sound with greater pitch perception and that the sound was clear. Others felt that the DCF strategy resulted in some background noise. The battery life of the PowerCel (Advanced Bionics, Sylmar, CA) Slim battery decreased from an average of $9 \mathrm{hr}$ with the clinical strategies to 1.5 to $4 \mathrm{hr}$ with the DCF strategy.

\section{DISCUSSION}

The DCF strategy showed promising results in this initial study, despite the large disparity in experience with the DCF

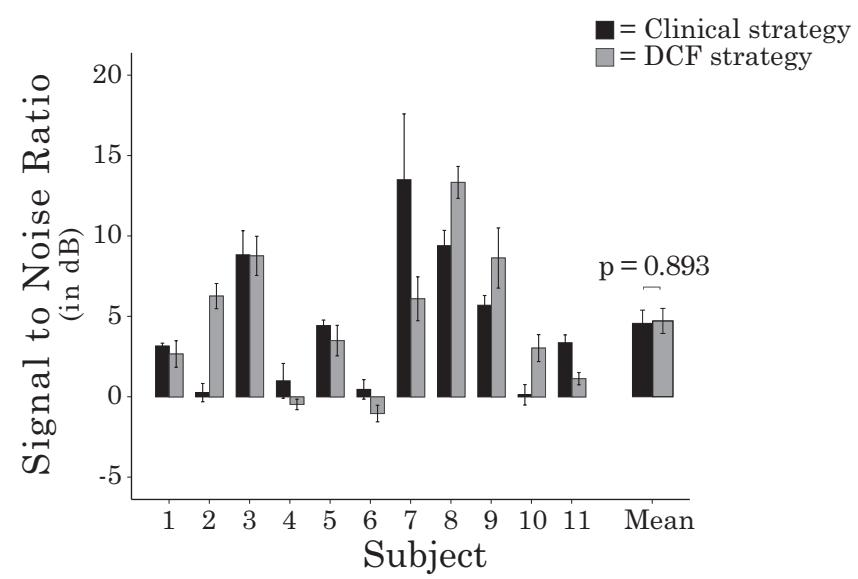

Fig. 6. Speech intelligibility in noise (MATRIX) with fixed speech at $65 \mathrm{~dB}$. SNR for which $50 \%$ of the words was repeated correctly. The error bars represent \pm 1 standard error of the mean. DCF indicates dynamic current focusing; SNR, speech-to-noise ratio. strategy versus the individuals' clinical strategies. Use of the DCF strategy improved spectral ripple discrimination by 1.34 RPO at lower loudness levels, which is a large improvement compared with reports in the literature using the same test. For example, Zhou (2017) found a 1.05 RPO improvement with an experimental strategy in which five high-threshold stimulation sites were deactivated, and Aronoff et al. (2016) showed that interleaved processors improve SMRT scores by 1.0 RPO. Moreover, a study where indiscriminable electrode contacts were deactivated in n-of-m strategies even found deteriorated SMRT scores (Vickers et al. 2016). The comparison of the DCF strategy results with other current focusing strategies is complex because often different spectral ripple measures are used. For example, Smith et al. (2013) found a 5.7 dB improvement in a spectral ripple phase discrimination experiment at 2.0 cycles/ octave when weighted TP stimulation was compared with MP stimulation. In our study, the most striking improvement was at $45 \mathrm{~dB}$, which was in accordance with our hypothesis, because the DCF strategy is set up such that higher levels of current focusing are achieved at lower loudness levels. Multiple studies have shown that the narrowing effect on current spread is negligible at $\sigma \leq 0.5$ (Bierer \& Middlebrooks 2002; Bonham \& Litvak 2008; Landsberger et al. 2012), while the mean degree of current focusing in this study was $0.88(0.8$ to 1.0$)$ at the T-level and $0.49(0.21$ to 0.74$)$ at the M-level. Thus, one could expect a greater benefit at lower loudness levels. Although most of the subjects benefited from current focusing across their entire dynamic range, some required fairly low $\sigma$ values to reach the M-level. The DCF strategy therefore did not enhance spatial selectivity at M-level, which explains why no significant difference was observed between DCF and MP stimulation in spectral resolution.

One potential disadvantage of current focusing techniques is that they require wider pulse widths, and thus lower stimulation rates, to reach sufficient loudness (Bonham \& Litvak 2008). This reduces temporal resolution and therefore speech perception. Although the stimulation rates decreased from 2550 pulses per second on average with the clinical strategy to 817 pulses per second with the DCF strategy, subjects performed equally well on the temporal modulation detection task using the two strategies. The DCF strategy was fitted without difficulties, and the subjects gave predominantly positive feedback on the sound quality. This is in accordance with the literature because previous studies also report positive results concerning the quality of sound with current focusing techniques (Landsberger et al. 2012; Padilla \& Landsberger 2016). Another disadvantage for the DCF strategy is the decrease in spectral channels (from 16 with HiRes or 120 theoretical channels with current steered strategies, to only 14 with DCF) that can be used because three physical electrode contacts are required to create one current focusing channel. Therefore, a smaller portion of the auditory nerve can be used for stimulation, possibly leading to a decrease in spectral resolution. Moreover, most subjects were clinically fitted with a speech coding strategy that uses current steering, which creates additional (virtual) spectral channels (Koch et al. 2007), while current steering was not implemented in the DCF strategy. Although many studies found a beneficial effect of the implementation of current steering (Firszt et al. 2009; Park et al. 2009), others were unable to find this improved performance (Berenstein et al. 2008; Donaldson et al. 2011; Snel-bongers et al. 2011). Nevertheless, the subjects in the present study 
may have benefited from current steering, that was only implemented in their clinical speech coding strategy.

Notably, acutely measured perception of speech-in-noise was as good with the DCF strategy as with the clinical strategy, even though the subjects had at least 9 months of experience with their clinical strategy but just a few hours of experience with the DCF strategy. Spectral ripple tests were added, as they are reported to be ideally suited for acute testing and correlate with long-term speech perception (Lawler et al. 2017), while speech tests need adaptation time. Thus, the significantly improved spectral ripple thresholds strengthen our conviction that the DCF strategy shows promise for improving the perception of speech-in-noise long term. While previous research did reveal significant correlations between SMRT scores and speech understanding (e.g., Lawler et al. 2017), we were not able to demonstrate this when the clinical $65 \mathrm{~dB}$ measures were used $[F(1,9)=1.8 ; p=0.211 ; R=0.41]$. This is probably due to the relatively small research group. Srinivasan et al. (2013) demonstrated improved speech understanding in an acute setting with pTP stimulation in six CI listeners after only $20 \mathrm{~min}$ of adaptation time. They found that SRTs were improved by 3 $\mathrm{dB}$ compared with an experimental MP strategy. However, they compared two strategies that were new to the subjects, whereas we compared the novel DCF strategy, to each subject's established clinical strategy.

Although the overall results with the DCF strategy were encouraging, not all subjects benefited from the novel loudness encoding strategy. Three subjects scored better on the spectral ripple test with the DCF strategy only at 45 or $65 \mathrm{~dB}$ but not at both levels. The results of the present study suggest that a change in loudness growth, from the clinical to the DCF strategy, could cause this lack of improved performance. The 3 subjects (S05, S09, and S10) who showed large discrepancies between the slopes of the loudness curves for the two strategies performed worse on the psychophysical tasks. Because a significant negative correlation was found between $\triangle \mathrm{AUC}$ and performance on the spectral ripple task (at $65 \mathrm{~dB}$ ), a post hoc analysis was performed leaving these 3 subjects (with the largest $\triangle$ AUCs) out, resulting in a greater improvement with the DCF strategy and a higher statistical significance $(p=0.006)$. While one would expect similar offsets of the loudness growth curves for the two speech coding strategies, remarkable deviations were found in some subjects, even across electrode contacts. The correlation between this $\Delta$ offset and spectral ripple scores at lower loudness levels was positive, although this was not statistically significant. It makes sense that subjects with negative $\Delta$ offsets performed worse at $45 \mathrm{~dB}$, as it could be that the sounds were inaudible, or at least very soft, at this loudness level. Altogether, this suggests that the way loudness growth is achieved could be of importance for CI performance. Nevertheless, previous research found only minor effects of loudness growth on speech performance (e.g., Fu \& Shannon 1998). This is consistent with our data, as we only found a detrimental effect on spectral ripple performance and not on speech perception. Moreover, it could be that longer adaptation to different loudness growth cancels out a detrimental effect. It is interesting that, S09 did not show unnatural loudness growth with the DCF strategy, but with the clinical Optima strategy. This subject had probably adapted to the aberrant loudness growth with the used clinical program and experienced difficulty adjusting to the (more regular) loudness growth of the DCF strategy. This observation highlights the beneficial effects of having a longer period of time to adapt to novel speech coding strategies, which might have resolved this issue. No clinical reasons were observed for the unexpected loudness growth with the clinical strategy for this subject (S09), such as an aberrant return pathway due to, for example, otosclerosis.

Bierer and Litvak (2016) suggested that especially poor performers benefit from strategies that reduce channel interactions, presumably because they suffer from more channel interaction in the first place. If there is a relatively poor electrode-neuron interface due to a large electrode-to-neuron distance (Bierer 2010; Bierer et al. 2015), the DCF strategy would theoretically greatly impact the overall performance. More laterally positioned electrodes benefit more from current focusing, as the efficacy of multipolar stimulation depends on interactions in the far field (Litvak et al. 2007a; Kalkman et al. 2015). This study mostly included subjects implanted with a HiFocus, 1J (Advanced Bionics, Sylmar, CA) electrode array, which is designed to be in an outer wall position, which in turn favors electrical field interaction. On the other hand, the study population comprised relatively good performers (consonant-vowel-consonant phoneme scores of $78 \%$ or more at $65 \mathrm{~dB}$ ), leaving relatively little room for improvement. The beneficial effects of the DCF strategy in a larger population of CI users with higher variation in performance might even be greater than in the present study.

If degeneration of the spiral ganglion cells underlies poor CI results, the beneficial effects of using a DCF strategy are likely to be less prominent. Several studies reported channelto-channel threshold variability across the electrode array to be highly correlated with poorer performance on speech tests (Pfingst et al. 2004; Pfingst \& Xu 2004; Bierer 2007; Long et al. 2014). High thresholds are believed to be caused, at least in part, by degeneration of the spiral ganglia or so-called dead regions, which presumably results in ineffective channels. As focused configuration leads to stimulation of a more localized region of the spiral ganglion, one could expect even more variability in focused stimulation mode (Bierer 2010), which could offset the beneficial effects of current focusing. Among the present study population, some subjects showed great variability in T-levels (most prominently S02 and S09). There are two possible solutions to this problem: (1) turn these electrode contacts off to improve speech intelligibility, as done by Bierer and Litvak (2016), or (2) switch the contact from TP to MP so that no auditory information is sent to ineffective channels. It was not possible to study possibly positive adaptations to the fitting, like switching off contacts with great variability, within the context of the present study because this would have introduced cofactors influencing the comparison of the two strategies.

We recently found that learning effects might interfere with the results of psychophysical measures used in this study (especially the SMRT and the MDT tests) (de Jong et al. 2017). However, this has only been shown in long-term studies, not in acute settings as in the present study (Drennan et al. 2010). In addition, the practice tests that were provided before the actual testing are likely to cancel out any minor acute learning effect (Lawler et al. 2017). Moreover, due to logistics, the DCF strategy was always tested last, at the end of a long day of testing. It seems likely that the decline in performance due to fatigue probably overcompensated for any potential learning effects. So we speculate that the gain in spectral resolution is underestimated in our study. Only S10, who was evaluated with the DCF 
strategy on a separate test day, may have benefitted from learning during the psychophysical tasks.

The main finding in this study was that the DCF strategy for loudness encoding significantly improved spectral resolution in an acute setting compared with the current clinically used stimulation strategies, in soft but not at higher presentation levels. As the battery life was considerably reduced, changes to the power scheme should be made to make the strategy suitable for at-home usage. A decrease in the T-level $\sigma$ to 0.8 and deactivation of ineffective electrode contacts could potentially increase battery life and therefore the clinical suitability of this novel loudness encoding strategy. Another potential solution to the high energy consumption of the DCF strategy might be to add parallel channels, as was done in the research of Langner et al. (2017). Although previous research in our clinic (Bonnet et al. 2012) showed that paired pulsatile stimulation might have a detrimental effect in MP mode, it has potential in current focused stimulation (Vellinga et al. 2017b). Also the use of n-of-m strategies might be a valid option to decrease battery consumption and is therefore of interest for future research. As benefits, particularly for speech intelligibility, of new speech coding strategies are generally greater after longer adaptation periods, the next step is to find out whether a greater improvement occurs over time in a take-home trial.

\section{CONCLUSIONS}

The present study showed that the DCF strategy gives better spectral resolution at lower loudness levels after only a few hours of adaptation to the strategy. Subjects had months of experience with the comparative MP speech coding strategies. Equal performance on speech and temporal modulation tests was found. Future research will reveal whether long-term usage of the DCF strategy also gives improved speech scores.

\section{ACKNOWLEDGMENTS}

This research was supported by nonrestrictive research funding from Advanced Bionics.

The authors have no conflicts of interest to declare.

Address for correspondence: Johan H. M. Frijns, ENT Department, Leiden University Medical Centre, P.O. Box 9600, 2300 RC Leiden, The Netherlands. E-mail: J.H.M.Frijns@lumc.nl

Received October 2, 2017; accepted March 13, 2018.

\section{REFERENCES}

Advanced Bionics (2012). HiResTM Optima Clinical Results (pp. 1-2). Sylmar, CA: Adv Bionics White Pap.

Aronoff, J. M., \& Landsberger, D. M. (2013). The development of a modified spectral ripple test. J Acoust Soc Am, 134, EL217-EL222.

Aronoff, J. M., Stelmach, J., Padilla, M., et al. (2016). Interleaved processors improve cochlear implant patients' spectral resolution. Ear Hear, 37, e85-e90.

Azadpour, M., \& McKay, C. M. (2012). A psychophysical method for measuring spatial resolution in cochlear implants. J Assoc Res Otolaryngol, 13, 145-157.

Berenstein, C. K., Mens, L. H., Mulder, J. J., et al. (2008). Current steering and current focusing in cochlear implants: Comparison of monopolar, tripolar, and virtual channel electrode configurations. Ear Hear, 29, 250-260.

Bierer, J. A. (2007). Threshold and channel interaction in cochlear implant users: Evaluation of the tripolar electrode configuration. $J$ Acoust Soc Am, 121, 1642-1653.
Bierer, J. A. (2010). Probing the electrode-neuron interface with focused cochlear implant stimulation. Trends Amplif, 14, 84-95.

Bierer, J. A., \& Faulkner, K. F. (2010). Identifying cochlear implant channels with poor electrode-neuron interface: Partial tripolar, single-channel thresholds and psychophysical tuning curves. Ear Hear, 31, 247-258.

Bierer, J. A., \& Litvak, L. (2016). Reducing channel interaction through cochlear implant programming may improve speech perception: Current focusing and channel deactivation. Trends Hear, 20, 1-12.

Bierer, J. A., \& Middlebrooks, J. C. (2002). Auditory cortical images of cochlear-implant stimuli: Dependence on electrode configuration. J Neurophysiol, 87, 478-492.

Bierer, J. A., Deeks, J. M., Billig, A. J., et al. (2015). Comparison of signal and gap-detection thresholds for focused and broad cochlear implant electrode configurations. J Assoc Res Otolaryngol, 16, 273-284.

Bonham, B. H., \& Litvak, L. M. (2008). Current focusing and steering: Modeling, physiology, and psychophysics. Hear Res, 242, 141-153.

Bonnet, R. M., Boermans, P. P., Avenarius, O. F., et al. (2012). Effects of pulse width, pulse rate and paired electrode stimulation on psychophysical measures of dynamic range and speech recognition in cochlear implants. Ear Hear, 33, 489-496.

Büchner, A., Lenarz, T., Boermans, P. P., et al. (2012). Benefits of the HiRes 120 coding strategy combined with the Harmony processor in an adult European multicentre study. Acta Otolaryngol, 132, 179-187.

de Jong, M. A. M., Briaire, J. J., Frijns, J. H. M. (2017). Learning effects in psychophysical tests of spectral and temporal resolution. Ear Hear, [Epub ahead of print].

Donaldson, G. S., Dawson, P. K., Borden, L. Z. (2011). Within-subjects comparison of the HiRes and Fidelity 120 speech processing strategies: Speech perception and its relation to place-pitch sensitivity. Ear Hear, $32,238-250$.

Drennan, W. R., Won, J. H., Nie, K., et al. (2010). Sensitivity of psychophysical measures to signal processor modifications in cochlear implant users. Hear Res, 262, 1-8.

Finley, C. C., \& Skinner, M. W. (2008). Role of electrode placement as a contributor to variability in cochlear implant outcomes. Otol Neurotol, $29,920-928$.

Firszt, J. B. (2003). HiResolution ${ }^{T M}$ Sound Processing (pp. 1-4). Sylmar, CA: Adv Bionics White Pap.

Firszt, J. B., Holden, L. K., Reeder, R. M., et al. (2009). Speech recognition in cochlear implant recipients: Comparison of standard HiRes and HiRes 120 sound processing. Otol Neurotol, 30, 146-152.

Francart, T., van Wieringen, A., Wouters, J. (2008). APEX 3: A multi-purpose test platform for auditory psychophysical experiments. J Neurosci Methods, 172, 283-293.

Fu, Q. J., \& Shannon, R. V. (1998). Effects of amplitude nonlinearity on phoneme recognition by cochlear implant users and normal-hearing listeners. J Acoust Soc Am, 104, 2570-2577.

Henry, B. A., \& Turner, C. W. (2003). The resolution of complex spectral patterns by cochlear implant and normal-hearing listeners. J Acoust Soc Am, 113, 2861-2873.

Holden, L. K., Firszt, J. B., Reeder, R. M., et al. (2016). Factors affecting outcomes in cochlear implant recipients implanted with a perimodiolar electrode array located in scala tympani. Otol Neurotol, 37, 1662-1668.

Houben, R., Koopman, J., Luts, H., et al. (2014). Development of a Dutch matrix sentence test to assess speech intelligibility in noise. Int J Audiol, $53,760-763$.

Jones, G. L., Drennan, W. R., Rubinstein, J. T. (2013). Relationship between channel interaction and spectral-ripple discrimination in cochlear implant users. J Acoust Soc Am, 133, 425-433.

Kalkman, R. K., Briaire, J. J., Frijns, J. H. (2015). Current focussing in cochlear implants: An analysis of neural recruitment in a computational model. Hear Res, 322, 89-98.

Khalili Moghaddam, G., Lovell, N. H., Wilke, R. G., et al. (2014). Performance optimization of current focusing and virtual electrode strategies in retinal implants. Comput Methods Programs Biomed, 117, 334-342.

Koch, D. B., Downing, M., Osberger, M. J., et al. (2007). Using current steering to increase spectral resolution in CII and HiRes $90 \mathrm{~K}$ users. Ear Hear, 28 (2 Suppl), 38S-41S.

Kral, A., Hartmann, R., Mortazavi, D., et al. (1998). Spatial resolution of cochlear implants: The electrical field and excitation of auditory afferents. Hear Res, 121, 11-28.

Landsberger, D. M., Padilla, M., Srinivasan, A. G. (2012). Reducing current spread using current focusing in cochlear implant users. Hear Res, $284,16-24$. 
Langner, F., Saoji, A. A., Büchner, A., et al. (2017). Adding simultaneous stimulating channels to reduce power consumption in cochlear implants. Hear Res, 345, 96-107.

Lawler, M., Yu, J., Aronoff, J. M. (2017). Comparison of the spectral-temporally modulated ripple test with the Arizona Biomedical Institute Sentence Test in cochlear implant users. Ear Hear, 1, 760-766.

Lazard, D. S., Vincent, C., Venail, F., et al. (2012). Pre-, per- and postoperative factors affecting performance of postlinguistically deaf adults using cochlear implants: A new conceptual model over time. PLoS One, 7, e48739.

Litvak, L. M., Spahr, A. J., Emadi, G. (2007a). Loudness growth observed under partially tripolar stimulation: Model and data from cochlear implant listeners. J Acoust Soc Am, 122, 967-981.

Litvak, L. M., Spahr, A. J., Saoji, A. A., et al. (2007b). Relationship between perception of spectral ripple and speech recognition in cochlear implant and vocoder listeners. J Acoust Soc Am, 122, 982-991.

Long, C. J., Holden, T. A., McClelland, G. H., et al. (2014). Examining the electro-neural interface of cochlear implant users using psychophysics, CT scans, and speech understanding. J Assoc Res Otolaryngol, 15 , 293-304.

Mens, L. H., \& Berenstein, C. K. (2005). Speech perception with monoand quadrupolar electrode configurations: A crossover study. Otol Neurotol, 26, 957-964.

Nadol, J. B. Jr, Young, Y. S., Glynn, R. J. (1989). Survival of spiral ganglion cells in profound sensorineural hearing loss: Implications for cochlear implantation. Ann Otol Rhinol Laryngol, 98, 411-416.

Nogueira, W., Litvak, L. M., Landsberger, D. M., et al. (2017). Loudness and pitch perception using dynamically compensated virtual channels. Hear Res, 344, 223-234.

Padilla, M., \& Landsberger, D. M. (2016). Reduction in spread of excitation from current focusing at multiple cochlear locations in cochlear implant users. Hear Res, 333, 98-107.

Park, H. J., Lee, S. C., Chun, Y. M., et al. (2009). HiRes with fidelity 120 benefit in native speakers of Korean. Cochlear Implants Int, 10(Suppl 1), 85-88.

Pfingst, B. E., \& Xu, L. (2004). Across-site variation in detection thresholds and maximum comfortable loudness levels for cochlear implants. $J$ Assoc Res Otolaryngol, 5, 11-24.

Pfingst, B. E., Xu, L., Thompson, C. S. (2004). Across-site threshold variation in cochlear implants: Relation to speech recognition. Audiol Neurootol, 9, 341-352.

Potts, L. G., Skinner, M. W., Gotter, B. D., et al. (2007). Relation between neural response telemetry thresholds, T- and C-levels, and loudness judgments in 12 adult nucleus 24 cochlear implant recipients. Ear Hear, $28,495-511$.

Smith, Z. M., Parkinson, W. S., Long, C. J. (2013). Multipolar current focusing increases spectral resolution in cochlear implants. Conf Proc IEEE Eng Med Biol Soc, 2013, 2796-2799.

Snel-bongers, J., Briaire, J. J., Vanpoucke, F. J., et al. (2011). Spread of excitation and channel interaction in single- and dual-electrode cochlear implant stimulation. Ear Hear, 32, 1-10.

Srinivasan, A. G., Padilla, M., Shannon, R. V., et al. (2013). Improving speech perception in noise with current focusing in cochlear implant users. Hear Res, 299, 29-36.

Srinivasan, A. G., Shannon, R. V., Landsberger, D. M. (2012). Improving virtual channel discrimination in a multi-channel context. Hear Res, 286, 19-29.

Vellinga, D., Briaire, J. J., van Meenen, D. M. P., et al. (2017a). Comparison of multipole stimulus configurations with respect to loudness and spread of excitation. Ear Hear, 38, 487-496.

Vellinga, D., Bruijn, S., Briaire, J. J., et al. (2017b). Reducing interaction in simultaneous paired stimulation with CI. PLoS One, 12, e0171071.

Vickers, D., Degun, A., Canas, A., et al. (2016). Deactivating cochlear implant electrodes based on pitch information for users of the ACE strategy. Adv Exp Med Biol, 894, 115-123.

Wardrop, P., Whinney, D., Rebscher, S. J., et al. (2005a). A temporal bone study of insertion trauma and intracochlear position of cochlear implant electrodes. I: Comparison of nucleus banded and nucleus contour electrodes. Hear Res, 203, 54-67.

Wardrop, P., Whinney, D., Rebscher, S. J., et al. (2005b). A temporal bone study of insertion trauma and intracochlear position of cochlear implant electrodes. II: Comparison of spiral clarion and HiFocus II electrodes. Hear Res, 203, 68-79.

Won, J. H., Drennan, W. R., Nie, K., et al. (2011). Acoustic temporal modulation detection and speech perception in cochlear implant listeners. $J$ Acoust Soc Am, 130, 376-388.

Won, J. H., Drennan, W. R., Rubinstein, J. T. (2007). Spectral-ripple resolution correlates with speech reception in noise in cochlear implant users. $J$ Assoc Res Otolaryngol, 8, 384-392.

Wu, C. C., \& Luo, X. (2013). Current steering with partial tripolar stimulation mode in cochlear implants. J Assoc Res Otolaryngol, 14, 213-231.

Zhou, N. (2017). Deactivating stimulation sites based on low-rate thresholds improves spectral ripple and speech reception thresholds in cochlear implant users. J Acoust Soc Am, 141, EL243-EL248.

Zhu, Z., Tang, Q., Zeng, F. G., et al. (2012). Cochlear-implant spatial selectivity with monopolar, bipolar and tripolar stimulation. Hear Res, 283, 45-58. 